\title{
Millimole Per Mole of Creatinine
}

National Cancer Institute

\section{Source}

National Cancer Institute. Millimole Per Mole of Creatinine. NCI Thesaurus. Code

C105511.

A unit of substance concentration defined as the concentration of millimoles of solute per mole of creatinine. 